\title{
Radicalización ideológico-política y terrorismo: un enfoque psicosocial
}

\section{Ideological-political radicalization and terrorism: a psychosocial approach}

\author{
Jorge Torres-Marín ${ }^{1}$, Ginés Navarro-Carrillo², Marcos Dono³, Humberto Manuel Trujillo ${ }^{4}$ \\ ${ }_{1}^{1}$ Departamento de Psicología Experimental y Fisiología del Comportamiento. Facultad de Psicología. \\ Universidad de Granada, España. ${ }^{2}$ Departamento de Psicología Social. Facultad de Psicología. \\ Universidad de Granada, España. ${ }^{3}$ Departamento de Psicología Social Básica y Metodología. \\ Facultad de Psicología. Universidad de Santiago de Compostela, España. ${ }^{4}$ Departamento de Metodología \\ de las Ciencias del Comportamiento. Facultad de Psicología. Universidad de Granada, España.
}

\begin{abstract}
Resumen
La radicalización ideológico-política y el terrorismo generan una serie de consecuencias desastrosas para el correcto funcionamiento de una sociedad. Actualmente, situaciones de convulsión social consecuencia de la crisis económica y migratoria, así como el terrorismo de corte ideológico-religioso, están ocasionando un aumento de la polarización y la radicalización ideológico-política. El planteamiento de intervenciones preventivas eficaces para atajar estos procesos requiere de un estudio sistemático y multidisciplinar en el que la psicología social debe ostentar un papel central. El objetivo del presente trabajo es hacer una propuesta comprensiva de corte conceptual que permita ordenar los procesos psicosociales que subyacen a la radicalización violenta de grupos con ideologías políticas extremistas. Concretamente, se pretende poner de manifiesto la idoneidad de analizar un conjunto de variables psicosociales que desempeñan un papel esencial en el equilibrio personal del individuo, en relación con el reclutamiento de terroristas, así como con el mantenimiento funcional de grupos radicalizados. Asimismo, y en respuesta a la necesidad de considerar nuevas perspectivas de investigación en el estudio del fenómeno terrorista, se examina el papel de la victimización y victimización competitiva, encontrándose suficientes argumentos teóricos como para recomendar la incorporación de dichos constructos en futuras investigaciones.
\end{abstract}

Palabras Clave: Radicalización; Terrorismo; Victimización; Victimización Competitiva; Enfoque Psicosocial.

\begin{abstract}
Ideological-political radicalization and terrorism generate a series of disastrous consequences for the correct functioning of society. Nowadays, polarization and ideological-political radicalization are on the rise. Factors contributing to this are situations of social upheaval like the migratory and economic crisis, as well as terrorism of an ideological-religious nature. The proposal of effective preventive interventions requires a multidisciplinary and systematic study of the phenomenon, in which social psychology is destined to play a central role. The aim of the current revision is to compile a comprehensive proposal of a conceptual nature, which will illustrate the psychosocial processes underlying violent radicalization of groups with extreme political ideologies. Specifically, the aim is to highlight the suitability of analyzing a set of psychosocial variables, which play an essential role in an individual's personal balance, related to terrorists' recruitment and the maintenance of extremist groups. Likewise, and as a response to the need to consider new research perspectives when studying the terrorism phenomenon, the part of victimization and competitive victimization in explaining terrorism is analyzed. The findings suggest enough theoretical links to encourage the integration of these constructs in future research.
\end{abstract}

Keywords: Radicalization; Terrorism; Victimization; Competitive Victimization; Psychosocial Approach.

Cómo citar este artículo: Torres-Marín, J., Navarro-Carrillo, G, Dono, M., y Trujillo, H. M. (2017). Radicalización ideológico-política y terrorismo: un enfoque psicosocial. Escritos de Psicología, 10, 134-146.

Correspondencia: Ginés Navarro Carrillo. Departamento de Psicología Social. Universidad de Granada. Campus Universitario de Cartuja s/n, 18071, Granada, España. .E-mail: ginesnc@ugr.es. E-mail de los co-autores: Jorge Torres Marín: jtorresmarin@ugr.es, Marcos Dono: marcos.dono019@gmail.com, Humberto M. Trujillo: humberto@ugr.es. 


\section{Introducción}

El papel decisivo de la violencia política en la trayectoria histórica de multitud de países representa un punto de convergencia entre ellos que ha precisado, precisa y precisará de la atención de la comunidad científica (Aróstegui, Callejo, \& Souto, 2000). El terrorismo en general, y concretamente el fundamentado en el radicalismo ideológico-político, juega un rol esencial en la seguridad y estabilidad de un Estado (Kruglanski, 2013). Por este motivo, la ciudadanía demanda la implementación de medidas eficaces en la lucha antiterrorista, en la prevención del reclutamiento de terroristas en contextos clave y, en definitiva, en la optimización de propuestas de carácter político orientadas a prevenir y erradicar esta preocupante lacra social (Moyano \& Trujillo, 2013; Skoczylis, 2015). Ante este escenario, es imprescindible que todas las disciplinas científicas implicadas en el estudio de esta temática aporten perspectivas y estrategias destinadas a la obtención de información precisa que permita la descripción, explicación y predicción rigurosa del comportamiento terrorista para, de este modo, poder prevenirlo (Horgan, 2006).

Cabe resaltar que hasta hace poco tiempo la psicología ha ostentado un papel poco significativo en el estudio sistemático del terrorismo, a pesar de ser una disciplina científica con un elevado potencial de rigurosidad para dicho fin (Horgan, 2006; Kruglanski, 2002). Han sido otras disciplinas, como la antropología, sociología, filosofía y politología, las que han trabajado -y continúan trabajando- en el objetivo de esclarecer la compleja naturaleza de este constructo (Raufer, 2006). No obstante, desde estas áreas de conocimiento, se ha priorizado el nivel de análisis macro o socio-estructural del terrorismo, focalizando la atención casi exclusivamente en la descripción de este fenómeno y casi nada en la explicación y predicción del mismo. Esto es, desde estas disciplinas no se ha profundizado en el estudio de los procesos psicosociales que hacen que una persona llegue a ser terrorista, derivándose de este enfoque información con menor rigor empírico y utilidad para llevar a cabo verdaderas acciones de prevención de la radicalización y el terrorismo (Horgan, 2006; Moyano \& Trujillo, 2013; Victoroff, 2005). Asimismo, desde un nivel de análisis micro o individual tampoco se ha conseguido contribuir de forma definitiva a la explicación del fenómeno, toda vez que la enorme heterogeneidad de motivaciones y personalidades que engloba una organización terrorista hace imposible detectar trastornos o rasgos de personalidad específicos que, por sí mismos, puedan desencadenar la emisión de la conducta terrorista (De la Corte, Kruglanski, de Miguel, Sabucedo, \& Díaz, 2007).

La naturaleza y complejidad de los procesos asociados a la radicalización violenta y al reclutamiento de terroristas (captación y adoctrinamiento), remite inequívocamente a la adopción de un enfoque meso o psico-grupal en el estudio del terrorismo (Moyano \& Trujillo, 2013). Cabe hacer explícito que cada vez es mayor el número de investigadores que deciden orientar el análisis del terrorismo desde un enfoque psicosocial, profundizando en el estudio de las interacciones existentes entre factores contextuales y personales que dan lugar a la emisión del comportamiento terrorista, y tratando de dilucidar posibles mecanismos y variables explicativas que ayuden a aclarar las causas del fenómeno (De la Corte et al., 2007; King \& Taylor, 2011). Por desgracia, el conocimiento científico actual sobre el comportamiento terrorista y los procesos que permiten la articulación, funcionamiento y supervivencia de organizaciones terroristas es relativamente limitado (Trujillo, 2009; Trujillo, Moyano, León, Valenzuela, \& González-Cabrera, 2006). Ante este escenario, la psicología en general y, especialmente, la psicología social en particular, representan una gran oportunidad para mejorar la comprensión de este objeto de estudio (Raufer, 2006), ya sea mediante el análisis de los procesos que hacen posible la aparición y mantenimiento del comportamiento terrorista, el estudio de la interacción entre el individuo y el colectivo ideológico radical, el análisis de las consecuencias de la actividad terrorista en todos los agentes implicados, o bien con la aportación de procedimientos técnicos y metodológicos que permitan obtener datos con mayor rigor científico (Horgan, 2006).

Así las cosas, el objetivo del presente trabajo es hacer una propuesta comprensiva de corte conceptual que permita ordenar los procesos psicosociales que subyacen a la radicalización violenta de grupos con ideologías políticas extremistas. Para optimizar la comprensión del tema, los contenidos del documento se organizan a través de una serie de apartados que permiten caer en la cuenta de la compleja casuística que subyace a la radicalización y el terrorismo político, así como de la relevancia de este fenómeno. Esto es, se pretende lo siguiente: (a) elucidar los conceptos de terrorismo, violencia y radicalización política; (b) analizar, siguiendo el modelo de vulnerabilidad a la captación y adoctrinamiento desarrollado por Trujillo y colaboradores (Moyano \& Trujillo, 2013; Trujillo, 2009; Trujillo et al., 2006;; Trujillo, León, Sevilla, \& González-Cabrera, 2010), las variables psicosociales implicadas en los procesos de reclutamiento de terroristas con intención política; (c) ahondar desde una perspectiva psicosocial en las variables que median en la supervivencia funcional de este tipo de organizaciones; (d) incorporar los procesos de victimización y victimización competitiva en el estudio psicosocial del terrorismo político; y (e) abordar posibles implicaciones psicosociales de la radicalización ideológico-política. 


\section{Aproximación conceptual al fenómeno terrorista: terrorismo, violencia y radicalización política}

El propósito de establecer una aproximación terminológica consensuada y precisa de qué se considera terrorismo o comportamiento terrorista, independientemente del matiz ideológico que lo fundamente, se ha revelado como un proceso capaz de generar confusión y controversia entre los miembros de la comunidad científica (Moyano \& Trujillo, 2013).

La complejidad inherente a la definición de terrorismo se debe no solo a la subjetividad asociada al constructo, sino también al estigma que lo acompaña y al que se somete a aquellas personas que son catalogadas como terroristas (Schmid, 2011). Cabe explicitar, además, que las múltiples conceptualizaciones propuestas sobre terrorismo parecen diferir sustancialmente en función de las disciplinas desde las cuales son formuladas (Schmid \& Jongman, 1988), lo que, de alguna manera, evidencia su carácter particularmente multifacético. No obstante, con el propósito de establecer una conceptualización integradora de terrorismo, Schmid (2011) propone una definición fundamentada en más de doscientas cincuenta definiciones académicas y gubernamentales:

El terrorismo se refiere por una parte a una doctrina acerca de la efectividad esperada de una forma o táctica especial de violencia política coercitiva que tiene como fin generar miedo; y, por otra parte, a la práctica conspirativa de violencia calculada, demostrativa y directa, ejercida sin ninguna constricción legal o moral, que tiene como objetivo principalmente civiles y no combatientes, y ejecutada por sus efectos propagandísticos y psicológicos en diversas audiencias y en grupos involucrados en el conflicto (Schmid, 2011, p.86).

En línea con lo señalado anteriormente, el terrorismo político podría definirse como el empleo de la violencia o de la amenaza de uso de la misma por parte de un individuo o de un colectivo con la finalidad de ejercer control sobre otras personas a través del miedo $\mathrm{y}$, de este modo, imponer un determinado posicionamiento ideológico dentro de un contexto político (Barreto \& Borja, 2007; Horgan, 2006).

Antes de comenzar a profundizar en el concepto de radicalización violenta, conviene dilucidar las particularidades de la violencia política y en qué condiciones aparece. El análisis pormenorizado de las manifestaciones de violencia política en diferentes contextos permite diferenciar una serie de rasgos comunes: a) suele estar integrada dentro de una estrategia mayor (está al servicio de esa causa); b) aparece ligada a otras iniciativas con objetivos idénticos o muy parecidos de carácter pacífico; y c) es menos frecuente que otras acciones o instrumentos de cambio político como la propaganda, la lucha electoral y parlamentaria, la concurrencia política o las manifestaciones (Aróstegui et al., 2000). En suma, se trata de una violencia de carácter instrumental que está al servicio de múltiples fines, a saber: promover una revolución o un golpe de estado, crear un estado de alarma entre la ciudadanía, obtener protagonismo político, reivindicar cambios socioestructurales, eliminar adversarios políticos, denunciar injusticias, etc. (Wieviorka, 1992). Por último, es conveniente indicar que la violencia y el terrorismo político no constituyen conceptos intercambiables, dado que el terrorismo no es más que una manifestación de un fenómeno más amplio como es la violencia política (Schmid, 2011).

Afortunadamente la totalidad de individuos que se identifican con una corriente ideológico-política extremista, no acabarán perpetrando actos terroristas. Dentro de la enorme heterogeneidad existente dentro de una población, habrá sectores de ésta que reconozcan la violencia como un medio adecuado para la obtención de beneficios afines a sus creencias, mientras que otros no (Trujillo et al., 2010). Por tanto, si "la gran mayoría de terrorista son radicales, pero la gran mayoría de radicales no son terroristas" (Trujillo et al., 2006, p. 293) -aludiendo el concepto de radical a una marcada inclinación, aplicable tanto a individuos como a colectivos, hacia actitudes y comportamientos extremistas en el marco de un determinado posicionamiento político (Borum, 2004; Tausch et al., 2011)- resulta innegable la importancia de identificar los factores y las relaciones funcionales que se dan entre ellos para que la predisposición al radicalismo desemboque en la emisión de conducta terrorista. Aunque el propósito de este trabajo no es profundizar en dichos condicionantes, tomando como referencia las aportaciones de Trujillo y colaboradores citadas anteriormente, se identifican factores sociales, internos y perceptivos. Los factores sociales o contextuales que determinan la violencia son la dispersión de la responsabilidad, desplazamiento de ésta bajo obediencia y desindividuación mediante la pérdida de identidad personal. Los factores internos derivados de un reajuste cognitivo que desinhiben la violencia son la deshumanización de la víctima, atribución de la culpa al propio agredido y justificación de la agresión bajo principios de orden moral superior. En último lugar, los factores perceptivos que legitiman la violencia son la desconfianza en el sistema legal, privación relativa, responsabilización de las víctimas e incremento de la autoeficacia para conseguir modificar las cosas. 
Tras la identificación de estos factores, resulta más sencillo obtener una mejor comprensión del significado de radicalización violenta. Así, este concepto haría referencia al resultado de un proceso de interacción entre numerosos aspectos, sociales e internos, a través del cual los individuos se adhieren a opiniones, puntos de vista e ideas de carácter extremista que pueden conducirles a cometer actos terroristas (Comisión Europea, 2002; Moyano, 2010). En esta misma línea, la radicalización violenta también ha sido conceptualizada como el proceso en el que la adquisición de las ideas radicales viene acompañada de la propensión a secundar o participar en actos violentos (Dalgaard-Nielsen, 2010). En cambio, otras propuestas terminológicas referidas a la radicalización violenta no contemplan la perpetración de actos terroristas como un elemento nuclear de dicho constructo, sino que, más bien, comprenden todas aquellas acciones que contravienen o amenazan los mecanismos de orden legal-democrático y que pueden alterar significativamente la convivencia en un Estado (AIVD, 2004). Estas divergencias entre las diferentes conceptualizaciones del proceso de radicalización, así como, en definitiva, entre los diferentes modelos teóricos dirigidos a su estudio, evidencian la necesidad de seguir profundizando en la comprensión psicológica de los procesos de radicalización (King \& Taylor, 2011).

Por último, cabe destacar que Kruglanski (2002) identificó algunas de las técnicas de influencia grupal usadas por organizaciones terroristas para catalizar este proceso de radicalización: a) aislamiento de la persona ante creencias alternativas a los ideales de la organización; b) deslegitimación y deshumanización de los posibles objetivos; c) tolerancia cero ante dudas o disentimientos; d) veneración por el líder; y e) reestructuración de la realidad social percibida.

\section{Análisis de los procesos de reclutamiento terrorista desde una perspectiva psicosocial}

En la búsqueda de un perfil global del terrorista, numerosas aproximaciones teóricas han tratado de establecer una relación causal entre la predisposición psicobiológica del individuo, o las variables socio-estructurales en las que se desarrolla, y la aparición del comportamiento terrorista (Moyano \& Trujillo, 2013). No obstante, la enorme heterogeneidad que presentan los terroristas desde un punto de vista psicológico, socio-demográfico, etc., hace que carezca de sentido contemplar una única teoría (Moyano, 2010; Post, 2001), ya que aun conociendo determinadas variables que pueden mediar en los procesos de captación y radicalización violenta, éstas no son suficientes para explicar -infiriendo causalidad categórica- la emisión del comportamiento terrorista (Trujillo, 2009).

Actualmente se está produciendo una revolución en el estudio del terrorismo, pues se ha pasado de la búsqueda de perfiles individuales a intentar determinar posibles variables que contribuyan al proceso de conversión terrorista. En este escenario, ha adquirido especial relevancia la perspectiva psicosocial (Barreto \& Borja, 2007). Este enfoque defiende que los actos terroristas no están determinados exclusivamente por variables sociales o psicológicas, sino por la interacción entre ambas a nivel intergrupal e intragrupal (De la Corte et al., 2007). Dentro de este ámbito, destaca el modelo desarrollado por Trujillo y colaboradores a lo largo de los últimos 15 años. Estos autores han ahondado en un conjunto de variables que desempeñan un papel esencial en el equilibrio personal del individuo, estableciendo un modelo descriptivo-explicativo sobre la vulnerabilidad de las personas a ser captadas (pre-radicalización) por organizaciones terroristas. A pesar de que este modelo estaba inicialmente centrado en el terrorismo de corte yihadista, resulta fácilmente extrapolable a otras problemáticas no sustentadas exclusivamente en un matiz ideológico-religioso. Más concretamente, podría aplicarse al desarrollo y mantenimiento de grupos con ideologías políticas extremistas.

Los estados de crisis personal y de pérdida de autonomía e integridad psicológica aumentan las probabilidades de que una persona caiga bajo las redes de organizaciones de carácter extremista (Trujillo et al., 2010). Por tanto, resulta innegable la importancia de identificar dichas variables con el propósito de implementar programas conducentes a la prevención del reclutamiento (Victoroff, 2005). El estado de vulnerabilidad para la captación de un individuo por una organización terrorista estaría relacionado, entre otras variables, con necesidades básicas no cubiertas, privación relativa, impulsividad y estrés, socavamiento de la motivación de logro personal, presencia de modelos incompetentes, baja robustez psicológica (baja resiliencia), déficits de apoyo socio-familiar, ausencia de modelos competentes y limitada preparación profesional (Moyano \& Trujillo, 2013).

Los entornos de marginación real, donde no sea posible satisfacer las necesidades básicas de una persona, o de marginación percibida, donde existe un sentimiento de privación relativa como consecuencia de la acción de un determinado estatus social o político que impide a los individuos desarrollar y manifestar sus creencias, creando déficits de reafirmación e identidad personal, actúan como indicadores de vulnerabilidad para los captadores de una organización terrorista (Trujillo, 2009; Trujillo et al., 2006). En el caso del terrorismo político europeo-occidental, con movimientos nacionalistas 
como el IRA o ETA a la cabeza, la marginación percibida por personas afines a ideologías reprimidas por el Estado, la percepción de falta de democracia o libertad civil, experiencias de injusticia social y la impresión de un reparto desigual o ilegítimo del poder, han actuado como algunos de los catalizadores más frecuentes en el proceso de captación (Aróstegui et al., 2000; Horgan, 2006; Tejerina, 1997). Es conveniente aclarar que no es necesario que el terrorista potencial haya tenido que experimentar de primera mano estas experiencias, toda vez que los procesos de socialización primaria y secundaria a través de los cuales los individuos asimilan los valores, creencias, roles sociales, ideas y, en definitiva, la idiosincrasia o cultura de un colectivo, son esenciales en el procesos de aprendizaje individual (De la Corte et al., 2007; Trujillo, 2009). Así pues, las personas que han sido socializadas en ambientes hostiles podrían desarrollar más fácilmente creencias y actitudes que les permitan reforzar la identidad con sus iguales (Trujillo et al., 2006).

Las situaciones de marginación anteriormente descritas tienen un efecto estresante sobre la persona (Berry, 1997), lo que desembocaría en un estado de interacción aversiva con el entorno que le rodea (Moyano, 2010). Asimismo, el estado de estrés crónico y agudo podría derivar en debilitamiento físico, provocando que el individuo sea más sugestionable (Moyano \& Trujillo, 2013). Bajo todas estas condiciones, la persona comienza a distanciarse de su contexto más cercano, pierde autoeficacia y duda sobre sus habilidades de afrontamiento, pasando a un estado de elevada alteración personal (irritabilidad, desequilibrio, etc.), con locus de control externo y con estilo atribucional interno; en otras palabras, con déficits de motivación hacia sus logros personales y, por consiguiente, con una mayor propensión a ser captada (Trujillo, 2009). Asimismo, el nivel de cualificación profesionaly la presencia de modelos competentes también pueden desempeñar un papel esencial en el perfil de vulnerabilidad (Moyano, 2010). En la misma línea, presentar un bajo nivel educativo se ha asociado al perfil del terrorista político en múltiples estudios (Horgan, 2006; Victoroff, 2005). Una baja cualificación académica suele estar vinculada a una menor fuente de ingresos e incertidumbre laboral, lo que podría derivar en sentimientos de marginación y frustración. Sin olvidar que durante la etapa educativa se adquieren gran parte de las habilidades necesarias para poder afrontar con éxito situaciones difíciles.

En síntesis, un individuo sin las habilidades de afrontamiento necesarias, ni apoyo social, ni la capacidad cognitiva para reorganizar pensamientos, presentaría dificultades para abandonar su situación de crisis personal, pudiendo ser influenciado por cualquier persona que le permita acabar con esta coyuntura (Moyano \& Trujillo, 2013). No obstante, cabe enfatizar que, si bien el modelo presentado anteriormente contempla una serie de factores que aumentan la vulnerabilidad de las personas a ser radicalizadas, la mera presencia de algunos de dichos factores no tiene por qué desencadenar per se dicho proceso, ya que esto sería simplificar en gran medida una realidad que abarca otros aspectos tales como la justificación de la acción violenta, desinhibidores de la agresión y elementos de disonancia cognitiva presentes en el perpetrador (Trujillo et al., 2006).

\section{Análisis del mantenimiento de grupos terroristas desde una perspectiva psicosocial}

Antes de profundizar en las variables psicosociales que contribuyen al mantenimiento funcional de una red o grupo terrorista, cabe señalar que uno de los aspectos fundamentales para la supervivencia de la organización es la obtención de los recursos económicos, tecnológicos, materiales y humanos necesarios para desarrollar su actividad, para lo que utilizarán técnicas como la amenaza, robo, coacción, secuestros, negocios ilegales, extorsión, etc. (De la Corte et al., 2007). Al margen de esto, para garantizar el desarrollo adecuado de este tipo de grupos se precisa de dos elementos clave: cohesión y lealtad (Trujillo et al., 2006).

En un conflicto intergrupal, la presencia de una amenaza o peligro externo, ya sea real o inducida ficticiamente por el aparato ideológico del endogrupo, optimiza la cohesión intragrupal (Barreto \& Borja, 2007). En este proceso de compactación del grupo, resulta esencial el papel que ejerce el líder ideológico de la organización. Según Trujillo (2009), entre otras posibles funciones del líder se destacan : a) facilitar la implantación de creencias positivas en el sistema y la forma de actuar del grupo, estableciendo pautas de planificación que fortalezcan a la propia organización; b) inspeccionar los canales de comunicación doctrinal, estratégica, operativa y táctica, es decir, controlar, dentro de su ámbito de acción, el aparato propagandístico y funcional del grupo; c) marcar a los miembros objetivos útiles para la causa; d) culpabilizar al exogrupo de los problemas internos que pueda tener la organización; y e) evitar periodos de inactividad que tornen al colectivo más vulnerable. De esta forma, centrar la atención en el exogrupo, considerado como un enemigo del endogrupo, difundiendo y, en ocasiones, exagerando de forma premeditada acciones de injusticia social, de represión violenta y de mala intencionalidad de los miembros de dicho exogrupo, favorece que los líderes ideológicos puedan legitimar el uso de la violencia contra el mismo (Barreto \& Borja, 2007). La legitimidad, tanto de la 
existencia de la organización terrorista como de la acción armada que lleva a cabo, es primordial para la supervivencia de ésta, ya que la no justificación de sus actos conllevaría la pérdida del apoyo social del movimiento y, por tanto, la posterior desaparición del mismo (Tejerina, 1997). En este sentido, el mismo autor sostiene que es imposible el mantenimiento funcional de un grupo extremista sin contar con el apoyo de un sector significativo de la sociedad, que ofrezca, entre otras cosas, apoyo logístico a la organización. Por este motivo, en las actuaciones contra los grupos terroristas se deben priorizar estrategias de intervención dirigidas a diezmar el apoyo social que reciben, pues esto dificulta los procesos de reclutamiento y mantenimiento operativo de las plantillas orgánicas que las conforman (Moyano \& Trujillo, 2013).

La segunda clave para garantizar la supervivencia y el adecuado funcionamiento del grupo terrorista es la lealtad, no tanto hacia las ideas que fundamentan a la organización, que también, como hacia los propios miembros que la componen (Trujillo, 2009). Además, con independencia del cariz ideológico-político, todos los grupos terroristas se caracterizan por el respeto incondicional hacia las reglas o directrices de la organización (Moyano, 2010). A mayor lealtad a los objetivos y las normas de la organización, mayor obediencia a los líderes e identificación con el grupo terrorista (De la Corte et al., 2007). En este sentido, el compromiso juega un papel central. Si el líder consigue que los miembros asuman un compromiso con la organización, la necesidad de mantenerse coherentes con dicho acuerdo les llevará a actuar en consecuencia (véase Cialdini, 1990).

Otro de los aspectos fundamentales para la perduración del grupo es la apología y disposición para la lucha por parte de los miembros. Los líderes coordinan una reformulación de la realidad percibida, a través de manuales, escrituras, noticias, etc., con la que se persigue propiciar el aislamiento, anular influencias externas, la pérdida de identidad personal y el convencimiento de la importancia de la acción individual para poder llevar a cabo estrategias para la consecución de un fin mayor, dotando de sentido a la acción armada y favoreciendo la cohesión endogrupal (Moyano \& Trujillo, 2013). Por último, tras la perpetración del acto terrorista "aparece por un lado la disonancia cognitiva, obligando al emisor del mismo a racionalizarlo y armonizar ideas con éste, y por otro, la aceptación y mejora de status en la organización y la entrada sin retorno en la clandestinidad" (Trujillo et al., 2006, p. 300).

\section{Victimización, victimización competitiva y terrorismo político}

Con el propósito de afrontar el estudio del terrorismo político, se hace necesario tomar en consideración nuevas perspectivas de investigación que contribuyan a comprender los factores asociados a la generación, legitimación y mantenimiento de la violencia terrorista. En la búsqueda de diferentes temas de investigación susceptibles de ser introducidos en este campo de conocimiento, el ámbito científico de la reconciliación intergrupal, cuyo objetivo se centra en promover una coexistencia pacífica entre grupos anteriormente enfrentados (Bar-Tal, 2000), ha puesto sobre la mesa la relevancia de los mecanismos individuales y grupales de la victimización y victimización competitiva en situaciones de conflicto. El análisis pormenorizado de la naturaleza de ambos constructos permite inferir una clara interconexión con variables y dimensiones referidas al paso de la radicalización no violenta hacia la emisión de conducta terrorista, así como con procesos adyacentes relacionados, la captación y mantenimiento funcional de organizaciones de esta índole.

Antes de continuar con la conceptualización de la victimización y victimización competitiva, es necesario señalar que el hecho de proponer la victimización competitiva como una explicación novedosa en el fenómeno del terrorismo requiere separarla de un concepto surgido previamente en este ámbito de estudio: la valoración asimétrica del sufrimiento (Sabucedo, Blanco, \& de la Corte, 2003). Si bien se trata de constructos diferenciados, la victimización competitiva y la valoración asimétrica del sufrimiento presentan suficientes similitudes en su semántica como para que sea pertinente una aclaración a este respecto. En líneas generales, aunque ambos procesos se centran en enfatizar el sufrimiento del endogrupo sobre aquel que experimenta el exogrupo, la diferencia central entre ambos radica en su naturaleza. Por un lado, la victimización competitiva supone un proceso grupal de carácter más autónomo, que surge en el seno del grupo sin dirección externa de carácter explícito, y que puede explicarse desde una perspectiva de necesidades identitarias grupales. Por otro lado, la valoración asimétrica del sufrimiento nace en el contexto del estudio de los discursos legitimadores de la violencia (Sabucedo et al., 2003). Constituye, por tanto, una narrativa elaborada de forma explícita, en contraste con el carácter más autónomo de la victimización competitiva, con una finalidad muy definida y tendente a justificar los actos terroristas. Todo ello implica, además, que se trata de una justificación del acto terrorista a posteriori, lo que evidenciaría su marcada influencia en el mantenimiento de la conducta terrorista y el apoyo ideológico hacia ésta, y no tanto en la emisión de la conducta violenta. 
Desde una perspectiva individual, la victimización hace referencia a la percepción que tiene el individuo de haber constituido el objeto, bien de manera momentánea, bien a lo largo de un periodo prolongado de tiempo, de comportamientos dañinos emitidos por una o más personas (Aquino \& Byron, 2002). Más específicamente, la victimización puede considerarse un estado mental duradero en el tiempo que genera actitudes (creencias, emociones y repertorios conductuales explícitos), y que acarrea consecuencias como sentimientos de desesperanza, autocompasión, ineficacia personal, baja autoestima, baja confianza, culpa y generalización hacia un locus de control externo (Bar-Tal, Chernyak-Hai, Schori, \& Gundar, 2009).

En lo que respecta a los mecanismos de orden grupal implicados en la victimización, la literatura científica informa sobre el papel de la memoria colectiva como un instrumento de perpetuación del sentimiento de daño e injusticia a través de generaciones, pudiendo dar lugar a estados de victimización ante eventos pertenecientes a un pasado distante (Bar-Tal et al., 2009; Noor, Shnabel, Hallabi, \& Nadler, 2012). Por otro lado, también se ha puesto de manifiesto la relevancia del fenómeno conocido como trauma escogido, según el cual el seno del grupo desarrolla una representación mental compartida sobre un suceso traumático del pasado que termina pasando a ser un elemento vertebrador de la identidad social del colectivo y sus miembros (Volkan, 2001).

La victimización competitiva hace referencia a la disputa entre dos grupos enfrentados por ser identificados como el receptor de más sufrimiento dentro de un conflicto (Noor, Brown, \& Prentice, 2008). El proceso de victimización no parece, a priori, presentar consecuencias positivas para el individuo, habida cuenta de que, como se ha citado con anterioridad, se relaciona con toda una serie de consecuencias negativas para el bienestar psicológico del mismo. Sin embargo, en el marco de un conflicto intergrupal, existen evidencias de que ambos colectivos tienden a centrarse en su propia condición de víctima (Nadler \& Saguy, 2003). Esta predisposición ha suscitado la exploración de mecanismos psicológicos susceptibles de explicar dicha realidad.

A nivel individual, en primer lugar cabe hacer referencia al concepto de etiquetamiento moral, proceso cognitivo relativo a la tendencia a clasificar a los distintos actores enfrentados en dos categorías excluyentes (víctima vs. agresor), siendo el rol de víctima indivisible y, por tanto, exclusivo para un único grupo (Noor et al., 2012). Así, y de acuerdo con la teoría de la identidad social (véase Tajfel 1981, 1982), los grupos tratarían de reafirmarse como vencedores en los procesos de comparación social que realizan con otros grupos al objeto de que su identidad social se vea reforzada. El hecho de atribuirse el papel de víctima se interpreta como una victoria con respecto al exogrupo, viéndose reforzada la idiosincrasia del colectivo a través de la asimilación de una serie de creencias, emociones y actitudes compartidas. Asimismo, la empatía vicaria podría llevar a que los individuos del endogrupo experimenten la victimización al presenciar o al ser informados del sufrimiento de otros miembros del grupo, llegando incluso a experimentarla aun cuando no hayan sufrido daños de forma directa (Bar-Tal et al., 2009), promoviéndose, así, el fortalecimiento de la cohesión grupal. En segundo lugar, también cabe mencionar el fenómeno conocido como brecha de significatividad, el cual hace referencia a la discrepancia entre víctimas y perpetradores a la hora de juzgar una transgresión. Las primeras juzgarían el acto como altamente severo e ilegítimo, mientras que los segundos lo definirían como legítimo, infravalorando la severidad del mismo (Baumeister, 1996; Noor et al., 2012).

Desde una perspectiva grupal, los sesgos de memoria ayudan a representar los diferentes sucesos en función de los propios objetivos y creencias, en lugar de representar fielmente lo ocurrido (Noor et al., 2012). Los sesgos en el procesamiento grupal del conflicto no se circunscriben solamente a sesgos de memoria, también se producen en torno a la valoración de los actos violentos del endogrupo, existiendo una tendencia a legitimar o presentar como más aceptables las transgresiones del mismo. El tratamiento típico de los conflictos intergrupales por parte de los medios de comunicación podría estar influyendo sobre estos sesgos, presentando a menudo estas situaciones como procesos de suma-cero, centrándose en el impacto negativo de las transgresiones en el momento presente sobre las causas del conflicto y/o aumentando la polarización al enfatizar las diferencias en lugar de las similitudes entre grupos (Lynch \& McGoldrick, 2005).

Además de los mecanismos psicológicos, es necesario no obviar los beneficios más tangibles que obtienen los grupos cuando logran el estatus de víctima, rol que va asociado generalmente a la obtención de un mayor apoyo por parte de la comunidad internacional; no solo ideológico, sino también en muchas ocasiones en forma de ayudas económicas (Bar-Tal et al., 2009).

Existen otras evidencias de carácter teórico-empírico relativas a la explicación de los mecanismos que subyacen a la voluntad de ostentar el papel de víctima principal en un conflicto. Entre ellas, cabe destacar las aportadas por el modelo basado en las necesidades (Nadler \& Shnabel, 2008). De acuerdo con este modelo, el grupo víctima y el grupo perpetrador (aunque dichos roles no suelen estar 
claramente definidos en situaciones de conflicto) presentan diferentes necesidades a satisfacer con respecto a su identidad. En lo tocante al grupo víctima, competir por este rol resulta acorde con sus necesidades de agencia, de manera que el hecho de que sea reconocido por terceras partes como tal implica un adeudamiento moral hacia el mismo que puede traducirse en influencia, así como en un mayor apoyo social y económico por parte de la comunidad internacional (Bar-Tal et al., 2009; Noor et al., 2012). Por su parte, el grupo perpetrador se presenta como víctima con el propósito de conseguir aceptación moral por parte de actores ajenos al conflicto. Así, se desencadenaría un proceso de victimización competitiva, ya que ostentar dicho papel supondría un factor diferencial en lo que se refiere al restablecimiento de las dimensiones deseadas.

Tras abordar los mecanismos causales de la victimización competitiva, y al objeto de mejorar la comprensión de la relación entre los beneficios de detentar el rol de víctima y el fenómeno terrorista de corte ideológico-político, es conveniente determinar las principales funciones de la victimización. Para ello, tomando como referencia el modelo de Bar-Tal et al. (2009), se establecen las siguientes: a) permite reducir el nivel de estrés y crisis personal producido por el conflicto a través de la explicación del mismo, generando una representación predecible de la realidad; b) se utiliza como justificación moral de las acciones que el grupo pueda llevar a cabo contra el exogrupo; c) acentúa las diferencias intergrupales, creando un imagen de superioridad con respecto al grupo rival; d) prepara al grupo para detectar y afrontar futuras amenazas o ataques, ayudando a identificar señales predictoras de alarma; e) aumenta la solidaridad entre los miembros del grupo en respuesta a una amenaza que ponga en riesgo la supervivencia del colectivo; y f) se emplea para obtener apoyo internacional, especialmente si se genera la percepción de ser el bando más débil del conflicto.

Llegados a este punto, ¿qué elementos vinculados a la victimización y victimización competitiva son susceptibles de introducirse en el estudio del terrorismo político desde una perspectiva psicosocial? En primer lugar, el terrorismo político se compone de una serie de procesos interconectados que van desde la vulnerabilidad a ser captado a la emisión de comportamientos terroristas, pasando por la radicalización violenta y el mantenimiento funcional de la organización. En este sentido, no sería aconsejable trazar una relación unívoca entre ciertos componentes de la victimización y/o victimización competitiva y una determinada fase del fenómeno terrorista. Por ejemplo, el proceso de victimización puede estar dirigido a propiciar tanto el reclutamiento del individuo como a facilitar el paso de la radicalización violenta a la emisión del comportamiento terrorista (véase Bar-Tal et al., 2009; Sabucedo, Rodríguez-Casal, \& Fernández-Fernández, 2002).

En lo que se refiere a la vulnerabilidad a la captación (pre-radicalización), resulta evidente que algunas de las consecuencias que se derivan de la victimización, como el socavamiento de la eficacia personal, la pérdida de autoestima y confianza, sentimientos de culpa, etc., podrían contribuir a acrecentar una percepción aversiva del entorno inmediato, desencadenando un estado de crisis personal que hace al individuo más proclive a ser reclutado por un colectivo terrorista (Bar-Tal et al., 2009; Trujillo et al., 2010). Este perfil de vulnerabilidad podría ser aprovechado por las personas dedicadas a la captación de potenciales miembros del colectivo a través de la utilización de estrategias manipulativas que permitan mitigar estas condiciones desagradables. En este sentido, la victimización, que se ha revelado como un mecanismo reductor del estrés asociado a las situaciones de conflicto, proporcionando una explicación y justificación del mismo (Bar-Tal et al., 2009), podría constituir uno de los elementos centrales del discurso de captación.

Tras la captación del individuo, el colectivo implementa una serie de técnicas grupales destinadas a exacerbar la radicalización del nuevo miembro (Kruglanski, 2002). Dichas técnicas persiguen, según Kruglanski, entre otras cosas, deslegitimar y deshumanizar a posibles objetivos, así como reajustar la realidad social percibida. Así pues, la victimización que, por un lado, presenta al grupo rival como perpetrador de un daño injusto e ilegítimo, atribuyéndole también la responsabilidad del inicio del conflicto y llegando incluso a deshumanizarlo (Bar-Tal et al., 2009) y, por otro , permite magnificar el conflicto actual y empeorar la visión del rival a través de fenómenos como el trauma escogido (Volkan, 2001), podría constituir una herramienta eficaz en la catalización del proceso de radicalización.

En lo que respecta a la perpetración de actos terroristas, se ha revelado la existencia de un vínculo entre el hecho de percibirse como víctima y la legitimación de las acciones violentas cometidas por el endogrupo (Noor et al., 2012; Sabucedo et al., 2003). Más específicamente, la victimización es un argumento retórico utilizado por diferentes grupos terroristas con la finalidad de deslegitimar al exogrupo y, de esta manera, reducir el posible conflicto moral derivado de ejercer la violencia contra el mismo, explicando sus propios actos violentos y justificándolos bajo el amparo del sufrimiento y las adversidades que sufre el endogrupo (Sabucedo et al., 2002). De hecho, en un estudio de Varela-Rey, Rodríguez-Carballeira y Martín-Peña (2013) dirigido a analizar el contenido de los discursos legitima- 
dores de la violencia del grupo terrorista ETA, se informó de la presencia de un elemento de discurso estrechamente relacionado con la victimización y que, a su vez, contenía dos subelementos: la violencia dañina del exogrupo y la violencia defensiva del endogrupo.

De igual modo, la victimización estaría vinculada con una mayor optimización del funcionamiento y mantenimiento del grupo. La victimización de un grupo genera solidaridad entre los miembros que lo componen, promoviendo una mayor cohesión grupal ante la amenaza real o percibida de un colectivo o rival que pone en riesgo su supervivencia funcional (Bar-Tal et al., 2009; Barreto \& Borja, 2007). Asimismo, Noor et al. (2012) también reivindican el papel de la victimización como elemento vertebrador del grupo que ayuda a evitar emociones grupales negativas y a buscar compensación. Por último, en aras de la supervivencia de la organización, es necesario contar con el respaldo de un amplio sector de la sociedad (Moyano \& Trujillo, 2013). Para ello, justificar la realización de actos violentos aludiendo a la injusticia cometida por el grupo perpetrador sobre el grupo víctima permitiría seguir contando con el apoyo social del movimiento (Bar-Tal et al., 2009; Tejerina, 1997).

En suma, todo lo expuesto anteriormente sobre las implicaciones de la victimización en los procesos de captación, radicalización y emisión de comportamiento terrorista, refuerza, en cierto modo, la perspectiva que defiende que los grupos podrían estar interesados en competir por ostentar el rol de víctima, pues esto contribuiría a incrementar el número de miembros reclutados, así como a favorecer una implicación más activa por parte de los mismos para con los objetivos del colectivo.

\section{Implicaciones de la radicalización ideológico-política de la población}

El análisis descriptivo de información difundida a través de medios de comunicación pone de relieve la discutible eficiencia en la lucha contra la proliferación de movimientos políticos de ideología extremista entre sectores específicos de la población (Barroso, 2014; Wheeler, 2012). A lo anterior, se une la profunda crisis económica y estructural que están sufriendo países como, por ejemplo, España, donde la incapacidad de los partidos políticos para potenciar medidas de fortalecimiento del estado del bienestar está acentuando el debilitamiento de la cohesión social (p.ej., Sánchez-Jiménez, 2010).

Ante esta coyuntura, y habida cuenta de su potencial relación con la emisión de conductas violentas y, en caso extremo, con la perpetración de actos terroristas -si se dan las condiciones adecuadas para ello- resulta innegable la necesidad de ahondar pormenorizada y rigurosamente en el estudio del fenómeno de radicalización ideológico-política, abordando entre otras cosas sus posibles implicaciones estructurales, sociales y psicológicas.

La radicalización ideológico-política constituye un elemento inherentemente vinculado a la polarización de la sociedad (véase Trujillo et al., 2010). El término polarización hace referencia a un fenómeno dinámico a través del cual un grupo asume una forma de interpretar la realidad, excluyendo la postura opuesta representada por otro colectivo (Moyano \& Trujillo, 2013). El contexto de una sociedad polarizada genera situaciones disfuncionales en diversos ámbitos. Una de sus manifestaciones más relevantes en la esfera sociopolítica tiene que ver con una mayor dificultad a la hora de establecer acuerdos para el uso de los recursos públicos (Alesina, Baqir, \& Easterly, 1997). Más específicamente, el enfrentamiento ostensible de intereses entre colectivos opuestos y, por consiguiente, de sus representantes políticos, se traduce en una gestión menos eficiente de los medios que el Estado posee para satisfacer las demandas de la ciudadanía (Gatica, 2012). Asimismo, la polarización social afecta a la normalización de la vida política, generando un clima de inseguridad, inestabilidad y crispación que no favorece el correcto funcionamiento de un Estado democrático (Keefer \& Knack, 2002).

Este distanciamiento político y social entre distintos sectores de la población sería, pues, uno de los principales catalizadores de la radicalización, especialmente entre aquellos colectivos más desfavorecidos (Trujillo et al., 2010). Por este motivo, se debería optimizar la gestión de ciertos recursos de los Estados (políticas de integración social, inserción laboral, reforma de las leyes sobre inmigración, etc.) al objeto de bloquear una de las probables antesalas a la radicalización y militancia terrorista (Moyano, 2010).

Los procesos psicológicos subyacentes al fenómeno de radicalización ideológico-política pueden abordarse desde diversas perspectivas teóricas. No obstante, la teoría de la identidad social (Tajfel, 1981, 1982) se ha consolidado como un eje central en el estudio de dicho fenómeno (Hogg, 2014; Schwartz, Dunkel, \& Waterman, 2009). Esta teoría propone que la concepción que los individuos tienen de sí mismos está en parte determinada por su pertenencia a ciertos grupos o categorías sociales y el valor que éstos otorgan a dicha pertenencia (véase Tajfel, 1981). La polarización social ligada al extremismo suele estar acompañada de la creación de prejuicios y estereotipos, los cuales resultan de los procesos de categorización que tienen lugar durante el conflicto intergrupal (Turner, 1999). De esta manera, podría perpetuarse una percepción negativa del exogrupo que, a su vez, favorecería la 
deshumanización de sus miembros, contribuyendo a legitimar, en última instancia, el empleo de la violencia (Barreto \& Borja, 2007; Trujillo, 2009). Otra perspectiva recientemente desarrollada, y conectada con los postulados de Tajel, que ha tratado de explicar las implicaciones psicológicas del proceso de radicalización ideológica es la teoría de la incertidumbre-identidad (Hogg, 2012). Dicha teoría establece que un estado saliente de incertidumbre personal lleva al individuo a identificarse con categorías sociales entitativas, esto es, colectivos fuertemente estructurados, distintivos, autoincluyentes y con ideologías extremistas, dado que éstos ofrecen una visión del yo más definida y una mayor orientación para su autoconcepto (véase Hogg, 2015). En este sentido, la pertenencia férrea a un grupo en una sociedad polarizada permite que el individuo no se encuentre en un estado de indefensión, sintiéndose protegido y amparado por los miembros de su endogrupo, mejorando así su percepción de autoeficacia y sus recursos de afrontamiento (Moyano, 2010; Trujillo, 2009). Este proceso de identificación extrema podría verse fortalecido por el aislamiento psicológico al que somete la organización al individuo, destruyendo paulatinamente su contacto con aquellas personas ajenas al colectivo extremista (familiares, amigos, etc.). A través de una serie de reajustes perceptivos inducidos por los líderes y adoctrinadores de la organización, se logra culpabilizar a dichas personas del distanciamiento personal producido (De la Corte et al., 2007).

Si bien el enfoque integral de corte psicosocial constituye el marco teórico desde el cual podría ser más factible inferir una explicación funcional del fenómeno de la radicalización violenta y el terrorismo, cabe resaltar también la importancia de ciertas aproximaciones teóricas más clásicas que actualmente están retomando relevancia en la explicación de estos fenómenos. Sea el caso de las teorías basadas en la frustración-agresión, privación relativa y opresión (Dollard, Doob, Miller, Mowrer, \& Sears, 1939; Gurr, 1970; Victoroff, 2005), y de otras de corte más individualista, como lo son las aproximaciones psicopatológicas, psicobiológicas y evolucionistas (Cooper, 1978; Hubbard, 1983; Victoroff, 2009), las cuales pueden aportar información complementaria a efectos de mejorar la comprensión del proceso de radicalización violenta y sus posibles consecuencias.

\section{Conclusiones}

Actualmente, el afrontamiento de situaciones sociales críticas como, por ejemplo, la acogida de los refugiados que huyen de las acciones violentas perpetradas por el autodenominado Estado Islámico, el aumento de la islamofobia y el auge de movimientos secesionistas, representan algunos de los principales focos de polarización ideológico-política en los países occidentales. La irrupción de grupos extremistas (p.ej., grupos contrarios a la cultura musulmana como Los Soldados de Odín en los países escandinavos, o Pegida en Alemania), junto con el fortalecimiento de opciones políticas con postulados extremistas, evidencian la necesidad de tomar medidas respecto a subsecuentes procesos de radicalización política y movimientos insurgentes y/o terroristas. En este sentido, el enfoque psicosocial constituye un modelo de análisis y actuación que enfatiza la importancia de factores psicológicos de corte personal y grupal relacionados con la radicalización, el reclutamiento de terroristas, la emisión de comportamientos violentos y la supervivencia funcional de organizaciones extremistas.

La innegable relevancia del fenómeno terrorista, unida a su compleja naturaleza (Raufer, 2006), obliga a explorar desde una óptica psicosocial nuevas temáticas de investigación que posibiliten una comprensión más completa de este constructo. Más específicamente, se ha propuesto que la victimización y victimización competitiva, tradicionalmente ligadas al ámbito de la reconciliación intergrupal (Noor et al., 2008), podrían contribuir a avanzar en la comprensión de los procesos de reclutamiento, la legitimación de la conducta violenta y los mecanismos que rigen el funcionamiento interno de los colectivos radicalizados y/o terroristas.

En definitiva, con este trabajo se ha pretendido hacer explícita la idoneidad de la perspectiva psicosocial en el estudio de la radicalización ideológico-política y el terrorismo. En este sentido, resultaría recomendable la implementación de nuevas investigaciones fundamentadas en este enfoque, dada su bondad para atender a las interacciones existentes entre diversos factores de corte personal y contextual, como marginalidad real o percibida, opresión, pérdida de confianza, intolerancia a la incertidumbre, cohesión y lealtad endogrupal, liderazgo, etc., susceptibles de explicar la adhesión a una ideología extremista (Moyano \& Trujillo, 2013).

Por último, es conveniente resaltar la importancia de destinar recursos, tiempo y atención investigadora al desarrollo de propuestas que engloben la cooperación de diferentes disciplinas de conocimiento, en aras de la optimización de las estrategias de prevención y afrontamiento de esta alarmante lacra social. 


\section{Referencias}

1. Alesina, A., Baqir, R., \& Easterly, W. (1999). Public goods and ethnic divisions. The Quarterly Journal of Economics, 114, 1243-1284. https://doi.org/10.1162/003355399556269

2. Aquino, K., \& Byron, K. (2002). Dominating interpersonal behavior and perceived victimization in groups: Evidence for a curvilinear relationship. Journal of Management, 28, 69-87. https://doi. org/10.1177/014920630202800105

3. Aróstegui, J., Calleja, E. G., \& Souto, S. (2000). La violencia política en la España del Siglo XX. Cuadernos de Historia Contemporánea, 22, 53-94.

4. AIVD.(2004). From Dawa to Jihad. The various threats from radical Islam to the democratic legal order. General Inteligence and Security Service.

5. Barreto, I., \& Borja, H. (2007). Violencia política: algunas consideraciones desde la psicología social. Revista Diversitas - Perspectivas en Psicología, 3, 109-119. https://doi.org/10.15332/ s1794-9998.2007.0001.07

6. Barroso, F. J. (16 de Marzo de 2014). El repunte de los ultras. El País. Recuperado de http://ccaa. elpais.com/ccaa/2014/03/15/madrid/1394901702_112540.html

7. Bar-Tal, D. (2000). From intractable conflict through conflict resolution to reconciliation: Psychological analysis. Political Psychology, 21, 351-365. https://doi.org/10.1111/0162-895X.00192

8. Bar-Tal, D., Chernyak-Hai, L., Schori, N., \& Gundar, A. (2009). A sense of self-perceived collective victimhood in intractable conflicts. International Review of the Red Cross, 91, 229-258. https://doi. org/10.1017/S1816383109990221

9. Baumeister, R. F. (1996). Evil: Inside human violence and cruelty. New York, NY: Henry Hold.

10. Berry. J. W. (1997). Immigration, acculturation and adaptation. Applied Psychology: An International Review, 46, 5-34. https://doi.org/10.1111/j.1464-0597.1997.tb01087.x

11. Borum, R. (2004). Psychology of terrorism. Tampa: University of South Florida.

12. Cialdini, R. B. (1990). Influencia, ciencia y práctica. Cuáles son los factores determinantes para que una persona diga sí a otra persona. Barcelona: Valls. S. A. Verdaguer.

13. Comisión Europea (2002, 13 de Junio). Decisión Marco 2002/465/JAI del Consejo, de 13 de Junio de 2002, sobre la lucha contra el terrorismo. Recuperado de http://www.consilium.europa. $\underline{\mathrm{eul}}$

14. Cooper, H. H. A. (1978). Psychopath as terrorist: A psychological perspective. Legal Medical Quarterly, 1, 16-32.

15. Dalgaard-Nielsen, A. (2010). Violent radicalization in Europe: What we know and what we do not now. Studies in Conflict \& Terrorism, 33, 797-814. https://doi.org/10.1080/1057610X.2010.501423

16. De la Corte, L., Kruglanski, A., de Miguel, J., Sabucedo, J. M., \& Díaz, D. (2007). Siete principios psicosociales para explicar el terrorismo. Psicothema, 19, 366-374.

17. Dollard, J., Doob, L. W., Miller, N. E., Mowrer, W., \& Sears, R. R. (1939). Frustration and aggression. New Haven, CT: Yale University Press. https://doi.org/10.1037/10022-000

18. Gatica, L. A. (2012). ¿Por qué el distanciamiento ideológico disminuye la provisión de bienes públicos? Una explicación basada en el empleo clientelar. Estudios de Economía, 39, 27-51.

19. Gurr, T. (1970). Why men rebel. Princeton, NJ: Princeton University Press.

20. Hogg, M. A. (2012). Uncertainty-identity theory. En P. A. M. Van Lange, A. W. Kruglanski, \& E. T. Higgins (Eds.), Handbook of theories of social psychology (Vol. 2, pp. 62-80). Thousand Oaks, CA: Sage. https://doi.org/10.4135/9781446249222.n29

21. Hogg, M. A. (2014). From uncertainty to extremism: Social categorization and identity processes. Current Directions in Psychological Science, 23, 338-342. https://doi. org/10.1177/0963721414540168

22. Hogg, M. A. (2015). To belong or not to belong: some self-conceptual and behavioural consequences of identity uncertainty / Pertenecer o no pertenecer: algunas consecuencias de la incertidumbre identitaria en el autoconcepto y en el comportamiento. Revista de Psicología Social, 30, 586-613. https://doi.org/10.1080/02134748.2015.1065090

23. Horgan, J. (2006). Psicología del Terrorismo. Cómo y por qué alguien se convierte en terrorista. Barcelona: Gedisa S. A.

24. Hubbard, D. G. (1983). The psychodynamics of terrorism. En Y. Alexander, \& T. Adeniran (Eds.), International Violence (pp. 45-52). Nueva York, NY: Praeger.

25. Keefer, P., \& Knack, S. (2002).Polarization, politics and property rights: Links between inequality and growth, Public Choice, 111, 127-54. https://doi.org/10.1023/A:1015168000336 
26. King, M., \& Taylor, D. M. (2011). The radicalization of homegrown jihadists: A review of theoretical models and social-psychological evidence. Terrorism and Political Violence, 23, 602-622. https:// doi.org/10.1080/09546553.2011.587064

27. Kruglanski, A. W. (2002). Inside the terrorist mind. Paper presented to the National Academy of Science. Washington, D. C.

28. Lynch, J., \& McGoldrick, A. (2005). Peace Journalism. Stroud: Hawthorn Press.

29. Moyano, M. (2010). Factores psicosociales contribuyentes a la radicalización islamista de jóvenes en España. Construcción de un instrumento de evaluación. Tesis Doctoral de Departamento de Psicología Social y Metodología de las Ciencias del Comportamiento. Universidad de Granada, Granada.

30. Moyano, M., \& Trujillo, H. M. (2013). Radicalización islamista y terrorismo. Claves psicosociales. Granada: Editorial Universidad de Granada.

31. Nadler, A., \& Saguy, T. (2003). Reconciliation between nations: Overcoming Emotional deterrents to ending conflicts between groups. En H. Langholtz \& C. E. Stout (Eds.), The psychology of diplomacy (pp. 29-46). New York, NY: Praeger.

32. Nadler, A, \& Shnabel, N. (2008) Instrumental and socioemotional paths to intergroup reconciliation and the needs-based model of socioemotional reconciliation. En A. Nadler, T. E. Malloy, \& J. D. Fisher (Eds.), The social psychology of intergroup reconciliation (pp. 37-56). New York, NY: Oxford University Press. https://doi.org/10.1093/acprof:oso/9780195300314.003.0003

33. Noor, M., Brown, J.R., \& Prentice, G. (2008). Precursors and mediators of intergroup reconciliation in Northern Ireland: A new model. British Journal of Social Psychology, 47, 481-495. https:// doi.org/10.1348/014466607X238751

34. Noor, M., Shnabel, N., Halabi, S., \& Nadler, A. (2012). When suffering begets suffering the psychology of competitive victimhood between adversarial groups in violent conflicts. Personality and Social Psychology Review, 16, 351-374. https://doi.org/10.1177/1088868312440048

35. Post, J.M. (15 November, 2001). The mind of the terrorist: individual and group psychology of terrorist behaviour. Testimony prepared for subcommittee on merging threats and capabilities, Senate Armed Services Committee, EEUU.

36. Raufer, X. (2006). Prólogo. En J. Horgan (Ed.), Psicología del Terrorismo. Cómo y por qué alguien se convierte en terrorista (pp. 9-13). Barcelona: Gedisa S. A.

37. Sabucedo, J. M., Blanco, A., \& De la Corte, L. (2003). Beliefs which legitimize political violence against the innocent. Psicothema, 15, 550-555.

38. Sabucedo, J. M., Rodríguez-Casal, M., \& Fernández-Fernández, C. (2002). Construcción del discurso legitimador del terrorismo. Psicothema, 14, 72-77.

39. Sánchez-Jiménez, J. (2010). Enfrentamiento partidista y polarización social: concepto y fases del proceso (1980-2000). Revista de Historia Contemporánea, 9, 35-51. https://doi.org/10.14198/ PASADO2010.9.03

40. Schmid, A. P. (2011). The Routledge handbook of terrorism research. New York, NY: Taylor \& Francis.

41. Schmid, A. P., \& Jongman, A. J. (1988). Political terrorism: A new guide to actors, authors, concepts, data bases, theories and literature. New Brunswick, NJ: Transaction Books.

42. Schwartz, S.J., Dunkel, C.S., \& Waterman, A.S. (2009). Terrorism: An identity theory perspective. Studies in Conflict \& Terrorism, 32, 537-559. https://doi.org/10.1080/10576100902888453

43. Skoczylis, J. J. (2015). The local prevention of terrorism: Strategy and practice in the fight against terrorism. London: Palgrave Macmillan. https://doi.org/10.1057/9781137499011

44. Tajfel, H. (1981). Human groups and social categories. Cambridge: Cambridge University Press.

45. Tajfel, H. (1982). Social identity and intergroup behavior. Cambridge: Cambridge University Press.

46. Tausch, N., Becker, J., Spears, R., Christ, O., Saab, R., Singh, P., et al. (2011). Explaining radical group behavior: Developing emotion and efficacy routes to normative and non-normative collective action. Journal of Personality and Social Psychology, 101, 129-148. https://doi.org/10.1037/ a0022728

47. Tejerina, B. (1997). Ciclo de protesta, violencia política y movimientos sociales en el País Vasco. Revista Internacional de Sociología, 16, 7-38.

48. Trujillo, H. M. (2009). Hacia una mejor comprensión psicológica del terrorismo: reclutamiento, ideología y violencia. Revista de Psicología Social, 24, 163-181. https://doi. org/10.1174/021347409788041426 
49. Trujillo. H. M., León, C., Sevilla, D., \& González-Cabrera, J. (2010). Riesgo de radicalización islamista en las mezquitas de una ciudad española. Psicología Conductual/Behavioral Psychology, 18, 434-440.

50. Trujillo, H. M., Moyano, M., León, C., Valenzuela, C. C., \& González-Cabrera, J. (2006). De la agresividad a la violencia terrorista: Historia de una patología psicosocial previsible (Parte II). Psicología Conductual/Behavioral Psychology, 14, 289-303.

51. Turner, J.C. (1999). Some current issues in research on social identity and self-categorisation theories. En N. Ellemers, R. Spears, \& B. Doosje (Eds.), Social identity: Context, commitment, content (pp. 6-34). Oxford: Blackwell.

52. Varela-Rey, A., Rodríguez-Carballeira, Á., \& Martín-Peña, J. (2013). Psychosocial analysis of ETA's violence legitimation discourse. Revista de Psicología Social, 28, 85-97. https://doi. org/10.1174/021347413804756050

53. Victoroff, J. (2005). The Mind of the Terrorist: A Review and Critique of Psychological Approaches. The Journal of Conflict Resolution, 49, 3-42. https://doi.org/10.1177/0022002704272040

54. Victoroff, J. (2009). Suicide terrorism and the biology of significance. Political Psychology, 30 , 397-400. https://doi.org/10.1111/j.1467-9221.2009.00704.x

55. Volkan, V. D. (2001). Transgenerational transmissions and chosen traumas: An aspect of largegroup identity. Group Analysis, 34, 79-97. https://doi.org/10.1177/05333160122077730

56. Wheeler, W. (17 de noviembre de 2012). Europe's new fascists. The New York Times. Recuperado de http://www.nytimes.com/2012/11/18/opinion/sunday/europes-new-fascists.html? $r=1$

57. Wieviorka, M. (1992). Terrorismo y violencia policía. Revista Internacional de Sociología, 2, 169178.

RECIBIDO: 8 de abril de 2016

MODIFICADO: 12 de enero de 2017

ACEPTADO: 19 de abril de 2017 